\title{
Zeta function regularization in de Sitter space: the Minkowski limit
}

\author{
Alan Chodos ${ }^{1}$ and András Kaiser ${ }^{2}$ \\ Center for Theoretical Physics, Sloane Physics Laboratory \\ Yale University, New Haven, Connecticut 06520-8120
}

\footnotetext{
${ }^{1}$ chodos@yalph2.physics.yale.edu

2 kaiser@hepunix.physics.yale.edu
} 


\begin{abstract}
:
We study an integral representation for the zeta function of the one-loop effective potential for a minimally coupled massive scalar field in D-dimensional de Sitter spacetime. By deforming the contour of integration we present it in a form suitable for letting the de Sitter radius tend to infinity, and we demonstrate explicitly for the case $\mathrm{D}=2$ that the quantities $\zeta(0)$ and $\zeta^{\prime}(0)$ have the appropriate Minkowski limits.
\end{abstract}

\title{
I. Introduction
}

In this paper we examine some properties of the effective potential for a minimally coupled scalar field in de Sitter spacetime. We regard this as a preliminary study with application to symmetry breaking effects in de Sitter space, which can have significant cosmological consequences [1]. These can be investigated if one also includes a potential for the scalar field.

Our particular concern in this work is to examine the regularization of the effective potential by the zeta-function method [2], and to show that after such regularization one can compute explicitly the limit of the potential as the de Sitter radius $\alpha$ tends to infinity, and recover thereby the Minkowski space result.

Of course de Sitter space itself becomes Minkowski space as the radius becomes infinite, and furthermore at the naive level the limit of the de Sitter zeta function $\zeta(s)$ goes over into the Minkowski one (see below). However in order to regularize the effective potential one must perform an analytic continuation of $\zeta$ to a neighborhood of $s=0$. After this this is done, it is no longer evident (or even necessarily true) that the zeta-function possesses the correct $\alpha \rightarrow \infty$ limit.

In other words, the process of analytic continuation and the $\alpha \rightarrow \infty$ limit may not commute with each other, and it is reassuring to check explicitly that the analytically-continued $\zeta(s)$ still possesses the expected Minkowski limit.

A simple example of what needs to be checked is provided by the series

$$
f(s ; a)=\sum_{n=0}^{\infty} e^{-(n+1) \alpha(s-2)}(-1)^{n}
$$

$(\alpha>0)$, which for $\Re s>2$ clearly obeys

$$
\lim _{\alpha \rightarrow \infty} f(s ; a)=0
$$

But $f(s ; a)$ possesses an analytic continuation in $s$

$$
f(s ; a)=\frac{1}{1+e^{\alpha(s-2)}}
$$

which, in a neighborhood of $s=0$ has the limit

$$
\lim _{\alpha \rightarrow \infty} f(s ; a)=1
$$

Let's take a look at the Minkowski zeta-function first. In $D$ dimensions, it is defined by

$$
\zeta(s)=\sum_{k} \frac{\mu^{2 s}}{\left(k^{2}+m^{2}\right)^{s}}
$$

where $\mu$ is a scale factor with dimensions of mass, that is included to make the dimension of $\zeta$ independent of $s$.

In Minkowski spacetime the sum is really continuous, so we can replace $\sum_{k}$ with $V d^{D} k /(2 \pi)^{D}(V$ is the volume of the spacetime) to transform it into an integral form

$$
\zeta(s)=V \int \frac{d^{D} k}{(2 \pi)^{D}} \frac{\mu^{2 s}}{\left(k^{2}+m^{2}\right)^{s}}
$$

or divide by $V$ to obtain the $\zeta$ density 


$$
\zeta(s)=\int \frac{d^{D} k}{(2 \pi)^{D}} \frac{\mu^{2 s}}{\left(k^{2}+m^{2}\right)^{s}} .
$$

\section{II. de Sitter zeta-function}

Proceeding to the de Sitter case we note that D-dimensional de Sitter spacetime can be embedded in a $\mathrm{D}+1$ dimensional Minkowski-space (we use signature $<+,-,-, \ldots,->$ ), where it is a $\mathrm{D}$ dimensional hypersurface determined by

$$
z^{0}-\sum_{i} z^{i^{2}}=-\alpha^{2}
$$

We use the following parametrization of this space

$$
\begin{aligned}
& z_{0}=\sinh \left(x_{0}\right) \\
& z_{1}=\cosh \left(x_{0}\right) \cos \left(x_{1}\right) \\
& z_{2}=\cosh \left(x_{0}\right) \sin \left(x_{1}\right) \cos \left(x_{2}\right)
\end{aligned}
$$

We start with the de Sitter action

$$
S=\int d^{D} x\left\{\partial_{\mu} \phi^{*} \partial^{\mu} \phi-m^{2}|\phi|^{2}\right\}
$$

Substituting $x_{0}=-i x_{4}$, we obtain the Euclidean action

$$
S=\int d^{D} x\left\{\partial_{\mu} \phi^{*} \partial^{\mu} \phi+m^{2}|\phi|^{2}\right\}
$$

The classical $\phi(x)$ field configurations form a vector space with the scalar product

$$
<\phi, \psi>=\int d x \phi^{*}(x) \psi(x)
$$

and the second variation of the action

$$
\frac{\delta^{2} S}{\delta \phi(x) \delta \phi^{*}(y)}=\left(\square_{E}+m^{2}\right) \delta(x-y)
$$

is similarly considered a matrix (bilinear operator on the vector space). The zeta function is defined with the eigenvalues of this matrix

$$
\zeta(s)=\sum_{i} \frac{\mu^{2 s}}{\lambda_{i}^{s}}
$$

where $\mu^{2 s}$ is included to get a dimensionless quantity. When we substitute $x_{0}=-i x_{4}, \cosh \left(x_{0}\right)$ transforms into $\cos \left(x_{4}\right)$, and $\sinh \left(x_{0}\right)$ into $-i \sin \left(x_{4}\right)$. This means that now we have

$$
\begin{aligned}
& z_{0}=-i \sin \left(x_{4}\right) \\
& z_{1}=\cos \left(x_{4}\right) \cos \left(x_{1}\right) \\
& z_{2}=\cos \left(x_{4}\right) \sin \left(x_{1}\right) \cos \left(x_{2}\right)
\end{aligned}
$$

which shows that Euclidean de Sitter space is a (series of) D-sphere(s), therefore the eigenfunctions (eigenvectors) of $\delta^{2} S / \delta \phi(x) \delta \phi(y)$ are generalized spherical functions, with the eigenvalue $\lambda=l(l+D-1) / \alpha^{2}+m^{2}$. The multiplicity of the modes with the same $l$ is [3] 


$$
\begin{aligned}
& \frac{\Gamma(D+l+1)}{\Gamma(D+1) \Gamma(l+1)}-\frac{\Gamma(D+l-1)}{\Gamma(D+1) \Gamma(l-1)} \\
& \text { so } \\
& \qquad(s)=\sum_{l}\left[\frac{\Gamma(D+l+1)}{\Gamma(D+1) \Gamma(l+1)}-\frac{\Gamma(D+l-1)}{\Gamma(D+1) \Gamma(l-1)}\right] \frac{\mu^{2 s}}{\left[\frac{l(l+D-1)}{\alpha^{2}}+m^{2}\right]^{s}} \\
& =\frac{\mu^{2 s} \alpha^{2 s}}{\Gamma(D+1)} \sum_{l}[(D+l)(D+l-1)-l(l-1)] \frac{\Gamma(D+l-1)}{\Gamma(l+1)} \frac{1}{\left[\left(l+\frac{D-1}{2}\right)^{2}-\beta^{2}\right]^{s}} \\
& \quad \text { where } \beta^{2}=-m^{2} \alpha^{2}+\left(\frac{D-1}{2}\right)^{2} \text {. } \\
& \text { Let's make a brief digression here, and check how the } \zeta \text {-density approaches the Minkowski case as } \alpha \rightarrow \infty \\
& \text { in other words the volume of the manifold } S^{D} \text {. } \\
& \text { of by the volume of de Sitter space, } \alpha^{D} V_{D}, \text { where } V_{D} \text { is the surface area of a } D+1 \text {-dimensional sphere, } \\
& \frac{1}{\alpha^{D} V_{D}} \zeta(s)=\frac{1}{\alpha^{D} V_{D}} \frac{1}{\Gamma(D+1)} \sum_{l}[(D+l)(D+l-1)-l(l-1)] \frac{\Gamma(D+l-1)}{\Gamma(l+1)} \frac{\mu^{2 s}}{\left[\frac{l(l+D-1)}{\alpha^{2}}+m^{2}\right]^{s}}
\end{aligned}
$$

Transform the sum over discrete values of $l$ into a continuous integral by substituting $l / \alpha \rightarrow k$ and $1 / \alpha \rightarrow$ $d k$. We also assume $l \gg 1$, yielding $(D+l)(D+l-1)-l(l-1) \rightarrow 2 D l=2 D k \alpha, \Gamma(D+l-1) / \Gamma(l+1) \rightarrow l^{D-2}$, and $l(l+D-1) / \alpha^{2} \rightarrow k^{2}$. This gives

$$
\frac{1}{\alpha^{D} V_{D}} \zeta(s)=\frac{2 D \mu^{2 s}}{\Gamma(D+1) V_{D}} \int \frac{k^{D-1} d k}{\left(k^{2}+m^{2}\right)^{s}}=\frac{2 D \mu^{2 s}}{\Gamma(D+1) V_{D}} \frac{1}{V_{D-1}} \int \frac{d^{D} k}{\left(k^{2}+m^{2}\right)^{s}} .
$$

Now substitute the value $V_{D}=2 \pi^{\frac{D+1}{2}} / \Gamma\left(\frac{D+1}{2}\right)$

$$
\frac{1}{\alpha^{D} V_{D}} \zeta(s)=\frac{2 D \mu^{2 s} \Gamma\left(\frac{D}{2}\right) \Gamma\left(\frac{D+1}{2}\right)}{\Gamma(D+1) 4 \pi^{D+1 / 2}} \int \frac{d^{D} k}{\left(k^{2}+m^{2}\right)^{s}}=\frac{\mu^{2 s} \Gamma\left(\frac{D+2}{2}\right) \Gamma\left(\frac{D+1}{2}\right)}{\Gamma(D+1) \pi^{D+1 / 2}} \int \frac{d^{D} k}{\left(k^{2}+m^{2}\right)^{s}}
$$

And finally use the identity $\Gamma(z) \Gamma(z+1 / 2) / \Gamma(2 z)=2^{1-2 z} \pi^{1 / 2}$

$$
\frac{1}{\alpha^{D} V_{D}} \zeta(s)=\frac{\mu^{2 s} 2^{-D}}{\pi^{D}} \int \frac{d^{D} k}{\left(k^{2}+m^{2}\right)^{s}}=\int \frac{d^{D} k}{(2 \pi)^{D}} \frac{\mu^{2 s}}{\left(k^{2}+m^{2}\right)^{s}},
$$

which is the Minkowski $\zeta$-density.

Now back to the de Sitter zeta-function, use the transformation [4]

$$
\frac{1}{\left[\alpha^{2}-\beta^{2}\right]^{\gamma}}=\frac{\sqrt{\pi}}{\Gamma(\gamma)} \int_{0}^{\infty}\left(\frac{t}{2 \beta}\right)^{\gamma-1 / 2} e^{-\alpha t} I_{\gamma-1 / 2}(\beta t) d t
$$

We get

$$
\zeta(s)=\frac{\mu^{2 s} \alpha^{2 s}}{\Gamma(D+1)} \sum_{l} D(D+2 l-1) \frac{\Gamma(D+l-1)}{\Gamma(l+1)} \frac{\sqrt{\pi}}{\Gamma(s)} \int_{0}^{\infty}\left(\frac{t}{2 \beta}\right)^{s-1 / 2} e^{-\left(l+\frac{D-1}{2}\right) t} I_{s-1 / 2}(\beta t) d t
$$

Concentrate on the terms involving $l$, and evaluate the sum 


$$
\begin{gathered}
\sum_{l} D(D+2 l-1) \frac{\Gamma(D+l-1)}{\Gamma(l+1)} e^{-\frac{1}{2}(D+2 l-1) t} \\
=-2 D \frac{\partial}{\partial t}\left[\sum_{l} \frac{\Gamma(D+l-1)}{\Gamma(l+1)} e^{-\frac{1}{2}(D+2 l-1) t}\right] \\
=-2 D \frac{\partial}{\partial t}\left[e^{-\frac{D-1}{2} t} \sum_{l} \frac{\Gamma(D+l-1)}{\Gamma(l+1)} e^{-l t}\right]=-2 D \frac{\partial}{\partial t}\left[e^{-\frac{D-1}{2} t} \frac{\Gamma(D-1)}{\left(1-e^{-t}\right)^{D-1}}\right] \\
=\Gamma(D+1) \frac{2 \cosh (t / 2)}{[2 \sinh (t / 2)]^{D}}
\end{gathered}
$$

Now use this result in the expression of the zeta-function

$$
\zeta(s)=\frac{\mu^{2 s} \alpha^{2 s}}{\Gamma(D+1)} \frac{\sqrt{\pi}}{\Gamma(s)} \int_{0}^{\infty}\left(\frac{t}{2 \beta}\right)^{s-1 / 2}\left[\Gamma(D+1) \frac{2 \cosh (t / 2)}{[2 \sinh (t / 2)]^{D}}\right] I_{s-1 / 2}(\beta t) d t
$$

With an appropriate correction factor, one can extend the contour of integration from $-\infty$ to $\infty$, integrating above the origin. We will need to introduce a branch cut somewhere in the complex plane between the origin and infinity; let this branch cut be on the negative imaginary axis (i.e. from the origin to $-i \infty)$, which corresponds to the contour passing the origin on the positive imaginary side to avoid the cut.

$$
\zeta(s)=\frac{\mu^{2 s} \alpha^{2 s} \sqrt{\pi}}{\Gamma(s)} \frac{1}{1-e^{i \pi(2 s-D)}} \int_{-\infty+i \Delta}^{\infty+i \Delta}\left(\frac{t}{2 \beta}\right)^{s-1 / 2} \frac{2 \cosh (t / 2)}{[2 \sinh (t / 2)]^{D}} I_{s-1 / 2}(\beta t) d t
$$

Here $\Delta$ is an arbitrary positive quantity, but less than $2 \pi$ to stay between the origin and the first pole of the integrand on the positive imaginary axis. This expression is the desired analytic continuation that is well-defined in a neighborhood of $s=0$.

\section{Deforming the contour}

Use an integral representation of the Bessel-function [5]

$$
\begin{gathered}
\zeta(s)=\frac{\mu^{2 s} \alpha^{2 s} \sqrt{\pi}}{\Gamma(s)} \frac{1}{1-e^{i \pi(2 s-D)}} \int_{-\infty+i \Delta}^{\infty+i \Delta}\left(\frac{t}{2 \beta}\right)^{s-1 / 2} \frac{2 \cosh (t / 2)}{[2 \sinh (t / 2)]^{D}} \\
{\left[\frac{1}{\sqrt{\pi} \Gamma(s)}\left(\frac{\beta t}{2}\right)^{s-1 / 2} \int_{-1}^{1} \frac{e^{\beta t u}}{\left(1-u^{2}\right)^{1-s}} d u\right] d t} \\
=\frac{\mu^{2 s} \alpha^{2 s}}{[\Gamma(s)]^{2}} \frac{1}{1-e^{i \pi(2 s-D)}} \int_{-\infty+i \Delta}^{\infty+i \Delta}\left(\frac{t}{2}\right)^{2 s-1} \frac{2 \cosh (t / 2)}{[2 \sinh (t / 2)]^{D}} \int_{-1}^{1} \frac{e^{\beta t u}}{\left(1-u^{2}\right)^{1-s}} d u d t
\end{gathered}
$$

We want to expand this expression in $s$ around $s=0$, so that we can deduce $\zeta$ and its first derivative from the result. These are the quantities that determine the effective potential.

First examine the integral over $\mathrm{u}$ :

$$
\int_{-1}^{1} \frac{e^{\beta t u}}{\left(1-u^{2}\right)^{1-s}} d u=\int_{0}^{1} \frac{e^{\beta t u}+e^{-\beta t u}}{\left(1-u^{2}\right)^{1-s}} d u=\int_{0}^{1} \frac{e^{\beta t(1-v)}+e^{-\beta t(1-v)}}{v^{1-s}(2-v)^{1-s}} d v
$$




$$
=e^{\beta t} \int_{0}^{1} \frac{e^{-\beta t v}}{v^{1-s}(2-v)^{1-s}} d v+(\beta t \leftarrow \rightarrow-\beta t)
$$

But

$$
e^{\beta t} \int_{0}^{1} \frac{e^{-\beta t v}}{v^{1-s}(2-v)^{1-s}} d v=e^{\beta t}\left\{\int_{0}^{1} \frac{1}{v^{1-s}(2-v)^{1-s}} d v-\int_{0}^{1} \frac{e^{-\beta t v}-1}{v^{1-s}(2-v)^{1-s}} d v\right\}
$$

The first term yields

$$
\frac{1}{2 s}+\ln 2+\mathrm{O}(s)
$$

The second term can be written as

$$
\begin{gathered}
\int_{0}^{1} \frac{1}{v^{1-s}(2-v)^{1-s}} \sum_{n=1}^{\infty} \frac{(-\beta t v)^{n}}{n !} d v \\
=\int_{0}^{1} \frac{1}{v(2-v)}\left[1+s \ln v+\mathrm{O}\left(s^{2}\right)\right]\left[1+s \ln (2-v)+\mathrm{O}\left(s^{2}\right)\right] \sum_{n=1}^{\infty} \frac{(-\beta t v)^{n}}{n !} d v
\end{gathered}
$$

Since we are looking only for the first two powers of $s$ in the expansion, and we have a $1 / 2 s$ term already, we will not need the terms proportional to $s$. Therefore we only need

$$
\int_{0}^{1} \frac{1}{v(2-v)} \sum_{n=1}^{\infty} \frac{(-\beta t v)^{n}}{n !} d v
$$

which gives

$$
\sum_{n=1}^{\infty} \frac{(-\beta t)^{n}}{n !}\left(2^{n-1} \ln 2-\sum_{m=1}^{n-1} \frac{2^{n-m-1}}{m}\right)
$$

So

$$
\begin{gathered}
\zeta(s)=\frac{\mu^{2 s} \alpha^{2 s}}{[\Gamma(s)]^{2}} \frac{1}{1-e^{i \pi(2 s-D)}} \int_{-\infty+i \Delta}^{\infty+i \Delta}\left(\frac{t}{2}\right)^{2 s-1} \frac{2 \cosh (t / 2)}{[2 \sinh (t / 2)]^{D}} \\
\left\{e^{\beta t}\left[\frac{1}{2 s}+\ln 2+\sum_{n=1}^{\infty} \frac{(-\beta t)^{n}}{n !}\left(2^{n-1} \ln 2-\sum_{m=1}^{n-1} \frac{2^{n-m-1}}{m}\right)+\mathrm{O}(s)\right]+(\beta t \leftarrow \rightarrow-\beta t)\right\}
\end{gathered}
$$

Here we have not yet expanded the terms outside the curly brackets in powers of $s$, we shall do it later. Evaluate the above expression for $m \alpha \gg 1$. This means $\beta=\sqrt{(D-1)^{2} / 4-m^{2} \alpha^{2}} \approx \pm i m \alpha$.

Let's find a way of closing the contour of integration at infinity in the upper or lower half plane, as appropriate for the different terms. First analyze the expression

$$
e^{i m \alpha t}\left[\frac{1}{2 s}+\ln 2+\sum_{n=1}^{\infty} \frac{(-i m \alpha t)^{n}}{n !}\left(2^{n-1} \ln 2-\sum_{m=1}^{n-1} \frac{2^{n-m-1}}{m}\right)+\mathrm{O}(s)\right]
$$

We can write $\ln 2$ as $\sum_{m=1}^{\infty} 2^{-m} / m$, and with this, 


$$
\begin{gathered}
\sum_{n=1}^{\infty} \frac{(-i m \alpha t)^{n}}{n !}\left(2^{n-1} \ln 2-\sum_{m=1}^{n-1} \frac{2^{n-m-1}}{m}\right)=\sum_{n=1}^{\infty} \frac{(-i m \alpha t)^{n}}{n !} \sum_{m=n}^{\infty} \frac{2^{n-m-1}}{m} \\
=\sum_{n=1}^{\infty} \frac{(-i m \alpha t)^{n}}{(n+1) !} \sum_{m=1}^{\infty} \frac{2^{-m}}{1+\frac{m-2}{n+1}}
\end{gathered}
$$

which clearly shows an $e^{-i m \alpha t} /(-i m \alpha t)$ asymptotic behavior for $|m \alpha t| \gg 1$. This combined with the $e^{i m \alpha t}$ in front yields $1 /(-i m \alpha t)$, which cancels with the similar term from $\beta t \leftarrow \rightarrow-\beta t$, so we can subtract it from the expression. Then the remaining expression will go to zero at least as fast as $1 /\left(m^{2} \alpha^{2} t^{2}\right)$. This allows as to close the contour for this term in the upper half plane. The integrand has poles in the upper half plane at $2 \pi i n, n=1,2 \ldots$. Applying the residue theorem, we obtain the integral as a sum

$$
\begin{gathered}
\frac{\mu^{2 s} \alpha^{2 s}}{[\Gamma(s)]^{2}} \frac{1}{1-e^{i \pi(2 s-D)}} \int_{-\infty+i \Delta}^{\infty+i \Delta}\left(\frac{t}{2}\right)^{2 s-1} \frac{2 \cosh (t / 2)}{[2 \sinh (t / 2)]^{D}} \\
e^{i m \alpha t}\left[-\frac{e^{-i m \alpha t}}{-i m \alpha t}+\frac{1}{2 s}+\ln 2+\sum_{n=1}^{\infty} \frac{(-i m \alpha t)^{n}}{n !}\left(2^{n-1} \ln 2-\sum_{m=1}^{n-1} \frac{2^{n-m-1}}{m}\right)+\mathrm{O}(s)\right] d t \\
=\left.\frac{\mu^{2 s} \alpha^{2 s}}{[\Gamma(s)]^{2}} \frac{1}{1-e^{i \pi(2 s-D)}} \sum_{n=1}^{\infty} 2 \pi i \frac{1}{\Gamma(D)} \frac{\partial^{D-1}}{\partial t^{D-1}}\right|_{t=2 \pi i n}\left\{\left(\frac{t}{2}\right)^{2 s-1} 2 \cosh (t / 2)\left(\frac{t-2 \pi i n}{2 \sinh (t / 2)}\right)^{D}\right. \\
\left.e^{i m \alpha t}\left[-\frac{e^{-i m \alpha t}}{-i m \alpha t}+\frac{1}{2 s}+\ln 2+\sum_{n=1}^{\infty} \frac{(-i m \alpha t)^{n}}{n !}\left(2^{n-1} \ln 2-\sum_{m=1}^{n-1} \frac{2^{n-m-1}}{m}\right)+\mathrm{O}(s)\right]\right\}
\end{gathered}
$$

From this form it can be seen that this contribution is finite, and it goes to zero with $m \alpha \rightarrow \infty$.

For the $e^{-i m \alpha t}$ term, we could similarly close the contour in the lower half plane, except that the integrand has a cut from the origin to $-i \infty$, because of the $(t / 2)^{2 s-1}$. So the curve that we use to close the contour must come back from $-i \infty$ to 0 in the positive real half plane, then go around the singularity at the origin anticlockwise, and go back to $-i \infty$ in the negative real half plane, before continuing the arc to $-\infty$. The complete contour constructed this way does not encircle any singularities, therefore the loop integral is zero, which means, that the contribution of the piece of contour along the cut cancels the contribution of the piece above the real axis, which is the integral that we want to determine (the two quarter-arcs from $\infty$ to $-i \infty$ and from $-i \infty$ to $-\infty$ contribute zero, similarly to the semi-arc in the upper half plane in the previous case). So our integral above the real axis will equal to the integral from $-i \infty$ to $-i \epsilon$ on the branch continuous with the left side of the cut, then a circle around zero clockwise with radius $\epsilon$, and then back from $-i \epsilon$ to $-i \infty$.

Now back to the previous expression, let's expand it in powers of $s$.

$$
\begin{gathered}
I(s)=\frac{\mu^{2 s} \alpha^{2 s}}{[\Gamma(s)]^{2}} \frac{1}{1-e^{i \pi(2 s-D)}} \int_{-\infty+i \Delta}^{\infty+i \Delta}\left(\frac{t}{2}\right)^{2 s-1} \frac{2 \cosh (t / 2)}{[2 \sinh (t / 2)]^{D}} \\
e^{-i m \alpha t}\left[-\frac{e^{i m \alpha t}}{i m \alpha t}+\frac{1}{2 s}+\ln 2+\sum_{n=1}^{\infty} \frac{(i m \alpha t)^{n}}{n !}\left(2^{n-1} \ln 2-\sum_{m=1}^{n-1} \frac{2^{n-m-1}}{m}\right)+\mathrm{O}(s)\right] d t
\end{gathered}
$$




$$
\begin{gathered}
=\frac{1}{-2 \pi i}\left[s+s^{2}\left(2 \ln (\mu \alpha)-2 \Gamma^{\prime}(1)+i \pi\right)+\mathrm{O}\left(s^{3}\right)\right] \int_{c}\left(\frac{2}{t}\left[1+2 s \ln \frac{t}{2}+\mathrm{O}\left(s^{2}\right)\right] \frac{2 \cosh (t / 2)}{[2 \sinh (t / 2)]^{D}}\right. \\
e^{-i m \alpha t}\left[-\frac{e^{i m \alpha t}}{i m \alpha t}+\frac{1}{2 s}+\ln 2+\sum_{n=1}^{\infty} \frac{(i m \alpha t)^{n}}{n !}\left(2^{n-1} \ln 2-\sum_{m=1}^{n-1} \frac{2^{n-m-1}}{m}\right)+\mathrm{O}(s)\right] d t
\end{gathered}
$$

\section{Evaluation of the integral}

Let's split the integral into the following terms:

$$
\begin{aligned}
& I_{1}=\int_{c} \frac{2}{t} \frac{2 \cosh (t / 2)}{[2 \sinh (t / 2)]^{D}} e^{-i m \alpha t} \frac{1}{2 s} d t \\
& I_{2}=\int_{c} \frac{2}{t} 2 s \ln \frac{t}{2} \frac{2 \cosh (t / 2)}{[2 \sinh (t / 2)]^{D}} e^{-i m \alpha t} \frac{1}{2 s} d t \\
& I_{3}=\int_{c} \frac{2}{t} \frac{2 \cosh (t / 2)}{[2 \sinh (t / 2)]^{D}} e^{-i m \alpha t} \frac{-e^{i m \alpha t}}{i m \alpha t} d t \\
& I_{4}=\int_{c} \frac{2}{t} \frac{2 \cosh (t / 2)}{[2 \sinh (t / 2)]^{D}} e^{-i m \alpha t} \ln 2 d t \\
& I_{5}=\int_{c} \frac{2}{t} \frac{2 \cosh (t / 2)}{[2 \sinh (t / 2)]^{D}} e^{-i m \alpha t} \sum_{n=1}^{\infty} \frac{(i m \alpha t)^{n}}{n !}\left(2^{n-1} \ln 2-\sum_{m=1}^{n-1} \frac{2^{n-m-1}}{m}\right) d t
\end{aligned}
$$

keeping only the two lowest-order terms in $s$. Only $I_{2}$ has a cut along the negative imaginary axis, so the other terms will decompose into the contributions of a series of poles along the negative imaginary axis. The contribution from all the poles except the origin can be expressed as

$$
\begin{aligned}
& \left.\frac{\mu^{2 s} \alpha^{2 s}}{[\Gamma(s)]^{2}} \frac{1}{1-e^{i \pi(2 s-D)}} \sum_{n=1}^{\infty} 2 \pi i \frac{1}{\Gamma(D)} \frac{\partial^{D-1}}{\partial t^{D-1}}\right|_{t=-2 \pi i n}\left\{\left(\frac{t}{2}\right)^{2 s-1} 2 \cosh (t / 2)\left(\frac{t-2 \pi i n}{2 \sinh (t / 2)}\right)^{D}\right. \\
& \left.e^{-i m \alpha t}\left[-\frac{e^{i m \alpha t}}{i m \alpha t}+\frac{1}{2 s}+\ln 2+\sum_{n=1}^{\infty} \frac{(i m \alpha t)^{n}}{n !}\left(2^{n-1} \ln 2-\sum_{m=1}^{n-1} \frac{2^{n-m-1}}{m}\right)+\mathrm{O}(s)\right]\right\}
\end{aligned}
$$

which is finite and goes to zero with $m \alpha \rightarrow \infty$, similarly to the contribution of the poles in the upper-half plane.

Now calculate the conribution from the pole at the origin for all but $I_{2}$. 


$$
\begin{aligned}
& I_{1}=\frac{1}{2 s} \int_{c} \frac{2}{t} \frac{2 \cosh (t / 2)}{[2 \sinh (t / 2)]^{D}} e^{-i m \alpha t} d t \\
& =\frac{1}{2 s} \int_{c} d t \frac{4}{t^{3}}\left[1+t^{2}\left(\frac{1}{8}-\frac{D}{24}\right)+\ldots\right]\left(1-i m \alpha t-\frac{1}{2} m^{2} \alpha^{2} t^{2}+\ldots\right) \\
& =\frac{2}{s}(-2 \pi i)\left(\frac{1}{24}-\frac{1}{2} m^{2} \alpha^{2}\right)=s^{-1} 2 \pi i\left(m^{2} \alpha^{2}-1 / 12\right) \\
& I_{3}=\int_{c} \frac{2}{t} \frac{2 \cosh (t / 2)}{[2 \sinh (t / 2)]^{D}} \frac{-1}{i m \alpha t} d t \\
& =-\frac{1}{i m \alpha} \int_{c} d t \frac{4}{t^{4}}\left[1+t^{2}\left(\frac{1}{8}-\frac{D}{24}\right)+\ldots\right]=0 \\
& I_{4}=\ln 2 \int_{c} \frac{2}{t} \frac{2 \cosh (t / 2)}{[2 \sinh (t / 2)]^{D}} e^{-i m \alpha t} d t \\
& =\ln 2 \int_{c} d t \frac{4}{t^{3}}\left[1+t^{2}\left(\frac{1}{8}-\frac{D}{24}\right)+\ldots\right]\left(1-i m \alpha t-\frac{1}{2} m^{2} \alpha^{2} t^{2}+\ldots\right) \\
& =4 \ln 2(-2 \pi i)\left(\frac{1}{24}-\frac{1}{2} m^{2} \alpha^{2}\right)=4 \pi i \ln 2\left(m^{2} \alpha^{2}-1 / 12\right) \\
& I_{5}=\int_{c} \frac{2}{t} \frac{2 \cosh (t / 2)}{[2 \sinh (t / 2)]^{D}} e^{-i m \alpha t} \sum_{n=1}^{\infty} \frac{(i m \alpha t)^{n}}{n !}\left(2^{n-1} \ln 2-\sum_{i=1}^{n-1} \frac{2^{n-i-1}}{i}\right) d t \\
& =\int_{c} d t \frac{4}{t^{3}}\left[1+t^{2}\left(\frac{1}{8}-\frac{D}{24}\right)+\ldots\right]\left(1-i m \alpha t-\frac{1}{2} m^{2} \alpha^{2} t^{2}+\ldots\right) \\
& \left(i m \alpha t \ln 2-\frac{1}{2} m^{2} \alpha^{2} t^{2}(2 \ln 2-1)-\frac{i}{6} m^{3} \alpha^{3} t^{3}\left(4 \ln 2-\frac{5}{2}\right)+\ldots\right) \\
& =4(-2 \pi i) 1 / 2 m^{2} \alpha^{2}=-4 \pi i m^{2} \alpha^{2}
\end{aligned}
$$

And now calculate $I_{2}$ on the contour that consists of a straight part from $-i \infty$ to $-i \epsilon$ on the branch of the ln function continuous with the left side of the cut, then a circle around the origin with radius $\epsilon$, and then another straight part from $-i \epsilon$ to $-i \infty$. First calculate the contribution of the straight parts. Since 
the direction of integration on the two sides of the cut is opposite, only the contribution of the discontinuity across the cut remains, which can be expressed as

$$
\begin{gathered}
\frac{1}{2 s} \int_{\epsilon}^{\infty} d x \frac{2}{x} 2 s(-2 \pi i) \frac{2 \cos (x / 2)}{[-2 i \sin (x / 2)]^{D}} e^{-m \alpha x} \\
=\int_{\epsilon}^{\infty} d x \frac{4}{x^{3}}(-2 \pi i) i^{D}\left[1-x^{2}\left(\frac{1}{8}-\frac{D}{24}\right)+\ldots\right] e^{-m \alpha x} \\
=\left[8 \pi i \frac{-1}{2 x^{2}} e^{-m \alpha x}\right]_{\epsilon}^{\infty}-8 \pi i \int_{\epsilon}^{\infty} d x \frac{-1}{2 x^{2}}(-m \alpha) e^{-m \alpha x}-8 \pi i \int_{\epsilon}^{\infty} d x \frac{1}{24 x} e^{-m \alpha x} \\
=4 \pi i \epsilon^{-2} e^{-m \alpha \epsilon}-\left[8 \pi i \frac{1}{2 x}(-m \alpha) e^{-m \alpha x}\right]_{\epsilon}^{\infty}+\int_{\epsilon}^{\infty} d x 4 \pi i \frac{1}{x}\left(m^{2} \alpha^{2}-1 / 12\right) e^{-m \alpha x} \\
+\left[4 \pi i \ln y\left(m^{2} \alpha^{2}-1 / 12\right) e^{-y}\right]_{\epsilon m \alpha}^{\infty}+4 \pi i \int_{\epsilon m \alpha}^{\infty} d y \ln y\left(m^{2} \alpha^{2}-1 / 12\right) e^{-y}
\end{gathered}
$$

Considering that $\int_{0}^{\infty} d y \ln y e^{-y}=\Gamma^{\prime}(1)$, we get

$$
\begin{gathered}
=4 \pi i \epsilon^{-2}(2+\epsilon m \alpha) e^{-m \alpha \epsilon}-4 \pi i \ln (\epsilon m \alpha)\left(m^{2} \alpha^{2}-1 / 12\right) e^{-\epsilon m \alpha} \\
+4 \pi i\left(m^{2} \alpha^{2}-1 / 12\right)\left(\Gamma^{\prime}(1)-\int_{0}^{\epsilon m \alpha} d y \ln y e^{-y}\right)
\end{gathered}
$$

Our procedure is to take $\epsilon \rightarrow 0$ before we let $m \alpha$ go to infinity. This means that we can expand this result in $\epsilon$ and discard all terms with positive exponents.

$$
\begin{gathered}
=4 \pi i \epsilon^{-2}(2+\epsilon m \alpha)\left(1-6 m \alpha+1 / 2 \epsilon^{2} m^{2} \alpha^{2}\right)-4 \pi i \ln (\epsilon m \alpha)\left(m^{2} \alpha^{2}-1 / 12\right) \\
+4 \pi i\left(m^{2} \alpha^{2}-1 / 12\right) \Gamma^{\prime}(1) \\
=4 \pi i \epsilon^{-2}-8 \pi i \epsilon^{-1} m \alpha+6 \pi i m^{2} \alpha^{2}-4 \pi i\left(m^{2} \alpha^{2}-1 / 12\right) \ln (\epsilon m \alpha)+4 \pi i\left(m^{2} \alpha^{2}-1 / 12\right) \Gamma^{\prime}(1)
\end{gathered}
$$

Now we must calculate the contribution of the circle around the origin. The radius of the circle is $\epsilon$, and $t$ can be expressed as $\epsilon e^{i \theta}$. This means that $\ln t=\ln \epsilon+i \theta, d t$ is equivalent to $i t d \theta$, and the integration around the circle from $-i \epsilon$ clockwise means that $\theta$ runs from $3 / 2 \pi$ to $-\pi / 2$. So for this part of the integral we have

$$
\begin{gathered}
\frac{1}{2 s} \int_{\frac{3}{2} \pi}^{-\frac{\pi}{2}} i t d \theta \frac{2}{t} 2 s\left(\ln \frac{\epsilon}{2}+i \theta\right) \frac{2 \cosh (t / 2)}{[2 \sinh (t / 2)]^{D}} e^{-i m \alpha t} \\
=2 i \ln \frac{\epsilon}{2} \int_{\frac{3}{2} \pi}^{-\frac{\pi}{2}} d \theta \frac{2 \cosh (t / 2)}{[2 \sinh (t / 2)]^{D}} e^{-i m \alpha t}-2 \int_{\frac{3}{2} \pi}^{-\frac{\pi}{2}} \theta d \theta \frac{2 \cosh (t / 2)}{[2 \sinh (t / 2)]^{D}} e^{-i m \alpha t}
\end{gathered}
$$




$$
\begin{aligned}
&= 2 i \ln \frac{\epsilon}{2} \int_{\frac{3}{2} \pi}^{-\frac{\pi}{2}} d \theta \frac{2}{t^{2}}\left[1+t^{2}\left(\frac{1}{8}-\frac{D}{24}\right)+\ldots\right]\left(1-i m \alpha t-\frac{1}{2} m^{2} \alpha^{2} t^{2}+\ldots\right) \\
&+2 \int_{-\frac{\pi}{2}}^{\frac{3}{2} \pi} \theta d \theta \frac{2}{t^{2}}\left[1+t^{2}\left(\frac{1}{8}-\frac{D}{24}\right)+\ldots\right]\left(1-i m \alpha t-\frac{1}{2} m^{2} \alpha^{2} t^{2}+\ldots\right) \\
&=4 i \ln \frac{\epsilon}{2}(-2 \pi)\left(\frac{1}{24}-\frac{1}{2} m^{2} \alpha^{2}\right)+4 \int_{-\frac{\pi}{2}}^{\frac{3}{2} \pi} \theta d \theta\left(t^{-2}-i m \alpha t^{-1}+\frac{1}{24}-\frac{1}{2} m^{2} \alpha^{2}+\ldots\right)
\end{aligned}
$$

Here we drop the terms that contain $t$ to a positive power, because on the circle $t=\epsilon e^{i \theta}$ for $t$, and those terms will vanish as $\epsilon \rightarrow 0$. Thus we have

$$
\begin{gathered}
-8 \pi i \ln \frac{\epsilon}{2}\left(\frac{1}{24}-\frac{1}{2} m^{2} \alpha^{2}\right)+4 \int_{-\frac{\pi}{2}}^{\frac{3}{2} \pi} \theta d \theta\left(\epsilon^{-2} e^{-2 i \theta}-i m \alpha \epsilon^{-1} e^{-i \theta}+\frac{1}{24}-\frac{1}{2} m^{2} \alpha^{2}\right) \\
=-8 \pi i \ln \frac{\epsilon}{2}\left(\frac{1}{24}-\frac{1}{2} m^{2} \alpha^{2}\right)+4\left[\theta \frac{1}{-2 i} \epsilon^{-2} e^{-2 i \theta}-\theta i m \alpha \frac{1}{-i} \epsilon^{-1} e^{-i \theta}+\left(\frac{1}{24}-\frac{1}{2} m^{2} \alpha^{2}\right) \frac{\theta^{2}}{2}\right]_{-\frac{\pi}{2}}^{\frac{3}{2} \pi} \\
-4 \int_{-\frac{\pi}{2}}^{\frac{3}{2} \pi} d \theta\left(\frac{1}{-2 i} \epsilon^{-2} e^{-2 i \theta}-i m \alpha \frac{1}{-i} \epsilon^{-1} e^{-i \theta}\right) \\
=-8 \pi i \ln \frac{\epsilon}{2}\left(\frac{1}{24}-\frac{1}{2} m^{2} \alpha^{2}\right)-4 \pi i \epsilon^{-2}+8 \pi i m \alpha \epsilon^{-1}+4 \pi^{2}\left(\frac{1}{24}-\frac{1}{2} m^{2} \alpha^{2}\right) \\
=-4 \pi i \epsilon^{-2}+8 \pi i \epsilon^{-1} m \alpha-2 \pi^{2}\left(m^{2} \alpha^{2}-1 / 12\right)+4 \pi i\left(m^{2} \alpha^{2}-1 / 12\right) \ln \frac{\epsilon}{2}
\end{gathered}
$$

To obtain $I(s)$, we assemble the contribution of the pole at the origin and the cut along the negative imaginary axis to $\zeta(s)$, to first order in $s$.

$$
I(s)=\frac{\mu^{2 s} \alpha^{2 s}}{[\Gamma(s)]^{2}} \frac{1}{1-e^{i \pi(2 s-D)}}\left\{I_{1}+I_{2}+I_{3}+I_{4}+I_{5}\right\}
$$




$$
\begin{aligned}
& =\frac{1}{-2 \pi i}\left[s+s^{2}\left(2 \ln (\mu \alpha)-2 \Gamma^{\prime}(1)+i \pi\right)+\mathrm{O}\left(s^{3}\right)\right] \\
& \left\{s^{-1} 2 \pi i\left(m^{2} \alpha^{2}-1 / 12\right)\right. \\
& +4 \pi i \epsilon^{-2}-8 \pi i \epsilon^{-1} m \alpha+6 \pi i m^{2} \alpha^{2}-4 \pi i\left(m^{2} \alpha^{2}-1 / 12\right) \ln (\epsilon m \alpha)+4 \pi i\left(m^{2} \alpha^{2}-1 / 12\right) \Gamma^{\prime}(1) \\
& -4 \pi i \epsilon^{-2}+8 \pi i \epsilon^{-1} m \alpha-2 \pi^{2}\left(m^{2} \alpha^{2}-1 / 12\right)+4 \pi i\left(m^{2} \alpha^{2}-1 / 12\right) \ln \frac{\epsilon}{2} \\
& +0 \\
& +4 \pi i \ln 2\left(m^{2} \alpha^{2}-1 / 12\right) \\
& \left.-4 \pi i m^{2} \alpha^{2}\right\} \\
& =\frac{1}{-2 \pi i}\left[s+s^{2}\left(2 \ln (\mu \alpha)-2 \Gamma^{\prime}(1)+i \pi\right)+\mathrm{O}\left(s^{3}\right)\right] \\
& \left\{s^{-1} 2 \pi i\left(m^{2} \alpha^{2}-1 / 12\right)+2 \pi i m^{2} \alpha^{2}-4 \pi i\left(m^{2} \alpha^{2}-1 / 12\right) \ln (m \alpha)\right. \\
& \left.+4 \pi i\left(m^{2} \alpha^{2}-1 / 12\right) \Gamma^{\prime}(1)-2 \pi^{2}\left(m^{2} \alpha^{2}-1 / 12\right)\right\} \\
& =\frac{1}{-2 \pi i}\left\{2 \pi i\left(m^{2} \alpha^{2}-1 / 12\right)\right. \\
& +s\left[\left(2 \ln (\mu \alpha)-2 \Gamma^{\prime}(1)+i \pi\right) 2 \pi i\left(m^{2} \alpha^{2}-1 / 12\right)\right. \\
& \left.+2 \pi i m^{2} \alpha^{2}-4 \pi i\left(m^{2} \alpha^{2}-1 / 12\right) \ln (m \alpha)+4 \pi i\left(m^{2} \alpha^{2}-1 / 12\right) \Gamma^{\prime}(1)-2 \pi^{2}\left(m^{2} \alpha^{2}-1 / 12\right)\right] \\
& \left.+\mathrm{O}\left(s^{2}\right)\right\} \\
& =-\left(m^{2} \alpha^{2}-1 / 12\right)+s\left[-m^{2} \alpha^{2}+2\left(m^{2} \alpha^{2}-1 / 12\right) \ln (m / \mu)\right]+\mathrm{O}\left(s^{2}\right)
\end{aligned}
$$

From this expression, keeping only the leading behavior in $(m \alpha)$, and dividing by $4 \pi \alpha^{2}$ (the volume of 2-D de Sitter space) to obtain the zeta-function density, we find

$$
\zeta(0)=-\frac{m^{2}}{4 \pi}
$$

and

$$
\zeta^{\prime}(0)=-\frac{m^{2}}{4 \pi}\left(1+\ln \frac{\mu^{2}}{m^{2}}\right) .
$$

For the Minkowski zeta-function density in 2-D, we have from eq. (1.7) 


$$
\zeta(s)=\int \frac{d^{D} k}{(2 \pi)^{D}} \frac{\mu^{2 s}}{\left(k^{2}+m^{2}\right)^{s}} \quad \cdot=\frac{m^{2}}{4 \pi}\left(\frac{\mu^{2}}{m^{2}}\right)^{s} \frac{1}{s-1}
$$

which gives precisely the values for $\zeta(0)$ and $\zeta^{\prime}(0)$ that we have obtained above.

\section{Conclusions}

In this paper we have, starting with the definition of the $\zeta$-function for a minimally coupled massive scalar field in D-dimensional de Sitter space, given an integral representation for it which is well-defined near $s=0$. We have then deformed the contour of integration so as to expose those terms which give the leading behavior as the de Sitter radius $\alpha$ tends to infinity.

In this limit, de Sitter space becomes Minkowski space, and we verify, specializing to the case $D=2$ for simplicity, that $\zeta(0)$ and $\zeta^{\prime}(0)$ tend to the expected Minkowski values. This is a property that is not at all evident from the original integral representation, and, as pointed out in the introduction, is a property that might have been lost in the process of analytic continuation. It is both reassuring and a non-trivial check of our computations that the Minkowski limit is in fact recovered after analytic continuation.

With these results in hand, one can consider the addition of a symmetry-breaking potential for the scalar field. One will then be able to study questions related to field quantization in de Sitter space in the presence of interactions that, in Minkowski space, would lead to symmetry breaking effects. In particular, one will be able to study the way in which particular time slicings may lead to symmetry restoration whereas others may allow the symmetry to remain broken [1]. Investigations along these lines are in progress. 


\section{Acknowledgements}

Research supported in part by DOE grant no. DE-AC02-ERO3075.

\section{References}

[1] B. Ratra, Phys. Rev. D 31, 1931 (1985)

B. Ratra, Phys. Rev. D 50 , 5252 (1994)

A. Kaiser and A. Chodos, Phys. Rev. D 53 , 787 (1996).

[2] J. S. Dowker and R. Critchley, Phys. Rev. D 13, 3224 (1976)

S. W. Hawking, Commun. Math. Phys. 55 , 133 (1977)

E. Elizalde, S. D. Odintsov, A. Romeo, A. A. Bytsenk and S. Zerbini, Zeta Regularization Techniques with Applications, World Scientific Publishing Co. Pre. Ltd., 1994.

[3] A. Chodos and E. Myers, Ann. Phys. 156 , 412 (1984)

[4] A. Erdélyi, W. Magnus, F. Oberhettinger and F. G. Tricomi, Higher Transcendental Functions - Bateman manuscript project, McGraw-Hill Book Company Inc., 1953.

[5] M. Abramowitz and I. A. Stegun, Handbook of mathematical functions, Dover books on advanced mathematics, 1972. 